\title{
Oligomerization Propensity and Flexibility of Yeast Frataxin Studied by X-ray Crystallography and Small-Angle X-ray Scattering
}

\section{Christopher A. G. Söderberg ${ }^{1} \dagger$, Alexander V. Shkumatov ${ }^{2} \dagger$, Sreekanth Rajan ${ }^{1}$, Oleksandr Gakh ${ }^{3}$, Dmitri I. Svergun ${ }^{2}$, Grazia Isaya $^{3 *}$ and Salam Al-Karadaghi ${ }^{1 *}$}

${ }^{1}$ Center for Molecular Protein Science, Institute for Chemistry and Chemical Engineering, Lund University, PO Box 124, SE-221 00 Lund, Sweden

${ }^{2}$ European Molecular Biology Laboratory, Hamburg Outstation, EMBL c/o DESY, Notkestraße 85, D-22603 Hamburg, Germany

${ }^{3}$ Department of Pediatric and Adolescent Medicine and Department of Biochemistry and Molecular Biology, Mayo Clinic, Rochester, MN 55905, USA

Received 27 July 2011; received in revised form 29 September 2011; accepted 19 October 2011 Available online 25 October 2011

\section{Edited by R. Huber}

Keywords: protein oligomerization; protein flexibility; metal chaperone; Friedreich's ataxia; neurodegenerative diseases
Frataxin is a mitochondrial protein with a central role in iron homeostasis. Defects in frataxin function lead to Friedreich's ataxia, a progressive neurodegenerative disease with childhood onset. The function of frataxin has been shown to be closely associated with its ability to form oligomeric species; however, the factors controlling oligomerization and the types of oligomers present in solution are a matter of debate. Using small-angle Xray scattering, we found that $\mathrm{Co}^{2+}$, glycerol, and a single amino acid substitution at the N-terminus, Y73A, facilitate oligomerization of yeast frataxin, resulting in a dynamic equilibrium between monomers, dimers, trimers, hexamers, and higher-order oligomers. Using X-ray crystallography, we found that $\mathrm{Co}^{2+}$ binds inside the channel at the 3-fold axis of the trimer, which suggests that the metal has an oligomer-stabilizing role. The results reveal the types of oligomers present in solution and support our earlier suggestions that the trimer is the main building block of yeast frataxin oligomers. They also indicate that different mechanisms may control oligomer stability and oligomerization in vivo.

(c) 2011 Elsevier Ltd. All rights reserved.
${ }^{*}$ Corresponding authors. E-mail addresses: isaya@mayo.edu; salam.al-karadaghi@mbfys.lu.se.

$\uparrow$ C.A.G.S. and A.V.S. contributed equally to this work.

Abbreviations used: SAXS, small-angle X-ray scattering; DLS, dynamic light scattering; PDB, Protein Data Bank; NSD, normalized spatial discrepancy; EOM, ensemble optimization method; EM, electron microscopy; DESY, Deutsches Elektronen-Synchrotron; GAJOE, Genetic Algorithm Judging Optimization of Ensembles.

\section{Introduction}

Iron is one of the most abundant elements on earth, and it is essential for most living organisms, despite the toxic effects associated with it. Thus, in an $\mathrm{O}_{2}$-containing environment and at physiological $\mathrm{pH}, \mathrm{Fe}^{2+}\left(6 \mathrm{H}_{2} \mathrm{O}\right)$ is readily oxidized to the acidic $\mathrm{Fe}^{3+}$ form, which rapidly produces insoluble $\mathrm{Fe}(\mathrm{OH})_{3}$. In addition, the one-electron oxidation of iron by oxygen produces superoxide that can in turn react to form hydrogen peroxide. Reaction of hydrogen peroxide with $\mathrm{Fe}^{2+}$ produces the highly reactive and toxic hydroxyl radical through the Fenton reaction. Hence, unligated iron carries a potential 
danger for cells and may have implication in a number of human diseases, which explains the need for a tight control of iron chemistry in organisms. ${ }^{1,2}$ Two proteins, ferritin and frataxin, are known to be central to the storage and detoxification of iron in cells. The importance of frataxin was first shown by the discovery that the autosomal recessive disease Friedreich's ataxia is linked to the presence of expanded GAA repeats in the first intron of the frataxin-encoding gene as well as to mutations in the gene sequence coding for the protein. ${ }^{3}$ Friedreich's ataxia affects approximately 1 in 40,000 individuals, with early onset-often before the age of 15 . Frataxin deficiency results in aberrations of cellular iron homeostasis and high production of reactive oxygen species, leading to a progressive neurodegenerative disease that is also characterized by cardiomyopathy and diabetes mellitus. ${ }^{4-6}$

Both ferritin and frataxin and their homologues have been implicated in the control of iron detoxification, which these proteins achieve by catalyzing the oxidation of ferrous iron to the ferric form and by storing it as a ferrihydrite biomineral within oligomeric species. ${ }^{7-11}$ In addition, the role of frataxin in iron homeostasis also includes iron delivery for ironsulfur cluster synthesis, heme biosynthesis, and aconitase repair. ${ }^{12-14}$ Frataxin species from humans and Saccharomyces cerevisiae (Yfh1) have been studied most extensively. They are expressed in the cytoplasm as 210-residue and 174-residue precursor polypeptides, respectively, with N-terminal mitochondrial targeting sequences. Proteolytic cleavage by mitochondrial processing peptidase results in the 52-174 mature form of yeast frataxin, while the human protein appears to exist as several variants with N-termini of different lengths (see below). ${ }^{15-18}$

The function of ferritin and frataxin has been strongly linked to their ability to form oligomeric species. In the case of ferritin, these oligomers are spontaneously assembled in cells, while for frataxin and its homologues, there appears to be variation in the propensity of the proteins from different sources to form oligomers and in the type of oligomers formed. ${ }^{19-2}$ In the presence of ferrous iron and oxygen, yeast frataxin has been shown to undergo stepwise assembly from monomers to larger oligomers that can contain up to 24 subunits, and even larger complexes. ${ }^{19}$ Higher-order oligomers can store up to $\sim 50-75$ iron atoms per subunit in 1- to 2-nm cores. ${ }^{11,19,22}$ The oligomeric species of yeast frataxin can be easily dissolved into monomers, for example, by the addition of reducing agents, ${ }^{19}$ suggesting that iron plays an active role in oligomer stabilization. In humans, at least four variants of frataxin have been isolated. The variants with the longer N-termini (56-210 and 42-210) can often assemble into larger structures in vivo and during expression in Escherichia coli. ${ }^{16,18}$ The large oligomers can be disassembled irreversibly into stable mono- mers by the addition of SDS. ${ }^{20}$ However, in contrast to the yeast Yfh1 protein, purified monomers of human frataxin do not appear to form oligomers in vitro, even in the presence of iron. ${ }^{16}$ This may indicate that assembly of the human protein requires additional assistance of some kind. Indeed, it has been shown that the Hsp70-type protein SSQ1 is required for Yfh1 to function in S. cerevisiae. ${ }^{23,24}$ The two other known variants of human frataxin, 81-210 and 78-210, have been found to be unable to adopt higher-order oligomeric states, ${ }^{18,25}$ indicating that residues $56-77$ at the $\mathrm{N}$-terminus of the protein are essential for oligomerization.

X-ray crystallographic studies of a variant of yeast frataxin in which amino acid Y73 was replaced by an alanine (Y73A) showed that this protein crystallized as a trimer, apparently stabilized by extensive interactions between the monomers around the 3-fold axis as well as by the $\mathrm{N}$-terminus, which bridged the monomers by forming additional interactions between them. ${ }^{26}$ Size-exclusion chromatography also showed that in contrast to wild-type Yfh1, which requires iron for assembly, the Y73A variant spontaneously assembled into larger oligomeric species that contained up to 24 monomers. ${ }^{26}$ In addition, singleparticle reconstruction studies of both iron-loaded and iron-free 24-meric particles clearly demonstrated that they were built up of trimeric units, ${ }^{22,26}$ suggesting that the trimer might be the main building block of larger frataxin oligomers. Interestingly, the arrangement of the functional features found in this structure, such as the position of the ferroxidation and iron mineralization sites and charge distribution inside and around the channel at the 3 -fold axis, has striking similarities to the arrangement of the corresponding features in ferritin, despite the absence of any evolutionary relationships at the amino acid sequence level or at the three-dimensional structural level. ${ }^{26}$

Given these data, the type of frataxin oligomers that exist in solution and the role of the different factors that control oligomer stability are poorly understood. In the current work, using a combination of smallangle X-ray scattering (SAXS), dynamic light scattering (DLS), and X-ray crystallography, we studied the oligomeric states of yeast frataxin in solution and the effects of glycerol, $\mathrm{Co}^{2+}$, and amino acid substitution at the $\mathrm{N}$-termini on these states. X-ray crystallography was used to reveal the cobalt binding site and its potential role in oligomer stabilization.

\section{Results}

\section{Structure of yeast frataxin in solution}

To gain some insight into the solution structure of wild-type yeast frataxin ( $\mathrm{Yfh} 1_{\mathrm{wt}}$; residues $\left.52-174\right)$ under different conditions, we used SAXS. The 
(a)

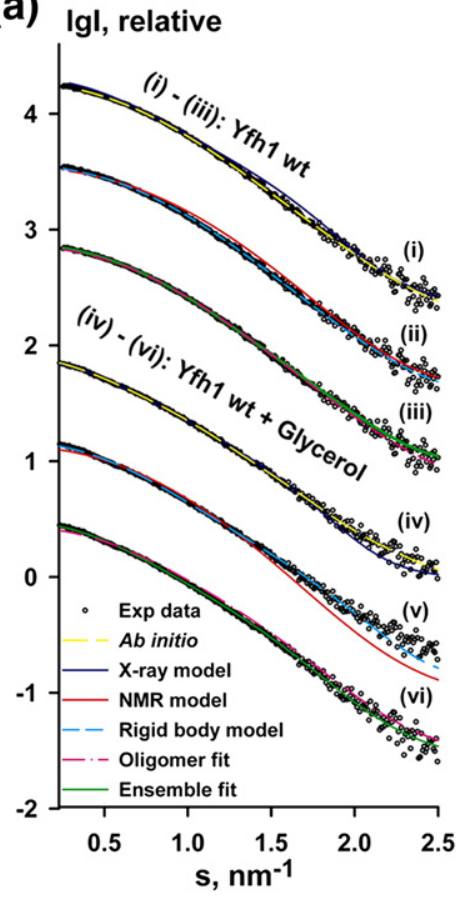

(b)
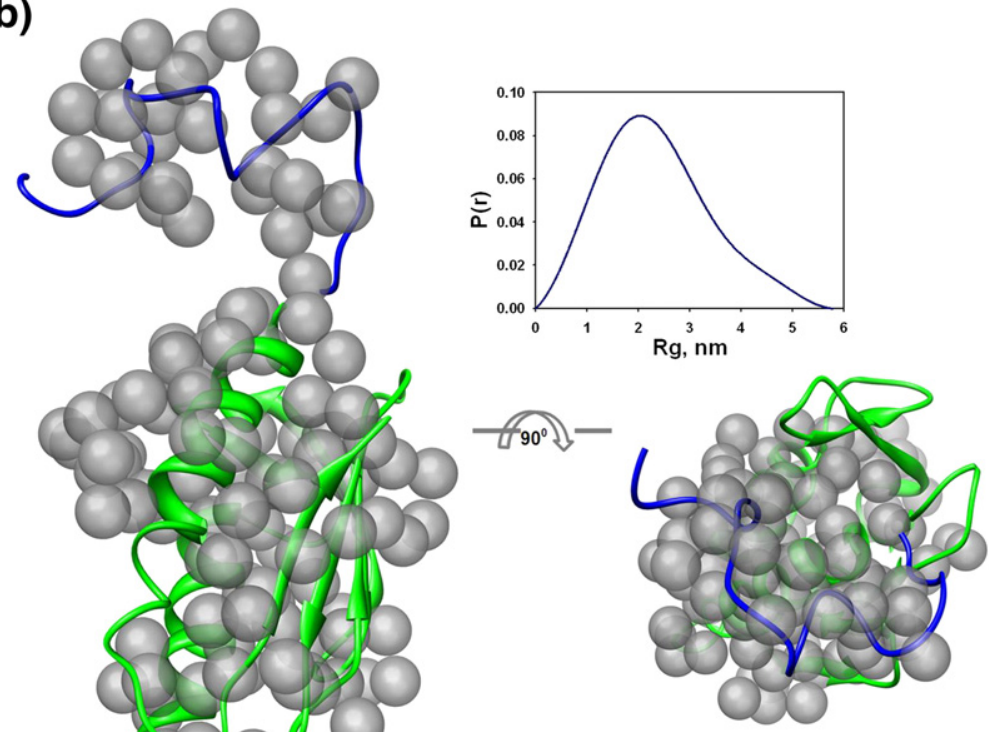

(c)

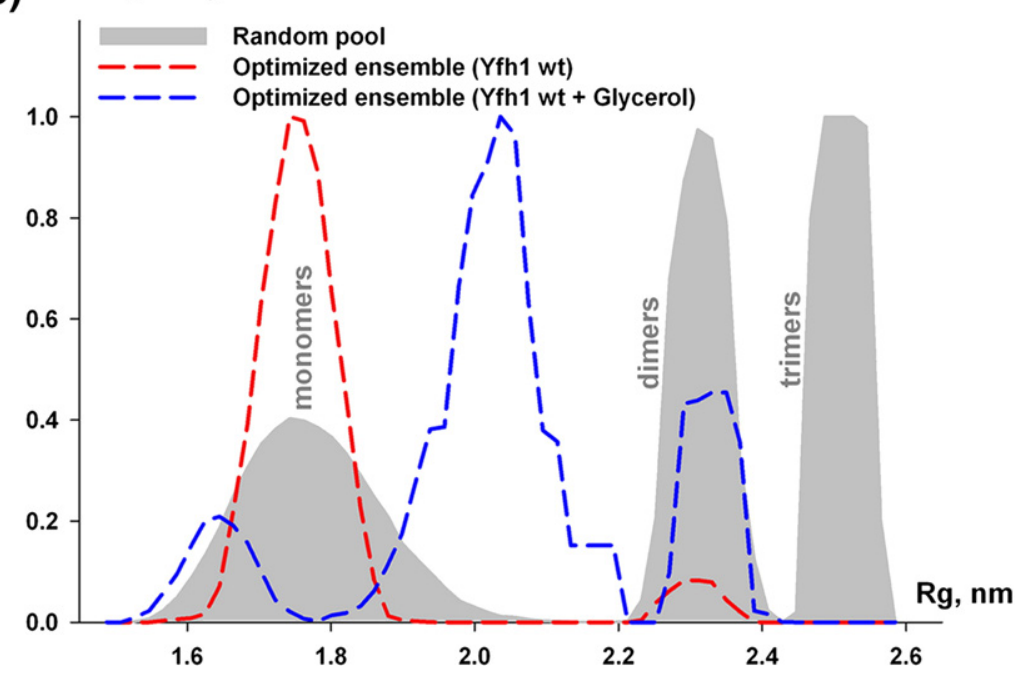

Fig. 1. SAXS measurements of yeast frataxin. (a) Experimental SAXS profiles (experimental data shown as black circles) for wild-type $\mathrm{Yfh} 1_{\mathrm{wt}}$ (i-iii) and $\mathrm{Yfh} 1_{\mathrm{wt}}+$ glycerol (iv-vi) were appropriately displaced along the logarithmic axis for better visualization and overlaid on the corresponding fits of the X-ray structure (i and iv), ab initio model (ii and v), lowestenergy NMR conformer (denoted NMR model on the figure, PDB ID: 2GA5), rigid-body model from OLIGOMER (denoted oligomer fit), and the EOM ensemble (denoted Ensemble fit, iii, and vi). Experimental SAXS data are shown to a maximal momentum transfer of $s=2.5 \mathrm{~nm}^{-1}$. See also Fig. S1b for plots of the Guinier region. (b) Superposition of the reference $a b$ initio model on the rigid-body model of $Y \mathrm{fh}_{1 \mathrm{wt}}$. Transparent gray beads represent the reference $a b$ initio model generated by GASBOR. The view on the right has been rotated by $90^{\circ}$ about the horizontal axis. The cartoon representation is used for the BUNCH model of frataxin (see the text). The blue part corresponds to the modeled $\mathrm{N}-$ terminus, and the green part corresponds to the available high-resolution structure used for modeling. The figures were prepared using the CHIMERA molecular graphics system. ${ }^{31}$ The inset shows the distance distribution function $p(r)$ used for $a b$ initio modeling. (c) Flexibility of frataxin in solution. $R_{\mathrm{g}}$ distribution for the random pool (6000 models) containing equal fractions of monomers, dimers, and trimers is shown by the area filled with gray. $R_{\mathrm{g}}$ distribution of optimized ensemble corresponding to wild-type Yfh1 (red broken line) and wild-type Yfh1+glycerol (blue broken line). 
advantages of using SAXS lie in the ability of this method to provide structural information for samples directly in solution. The method tolerates a wide range of protein or protein complex sizes ${ }^{27}$ and has successfully been used for modeling of proteins with rather short flexible parts, for example, hinge regions in multidomain proteins, ${ }^{28}$ as well as natively unfolded proteins. ${ }^{29,30}$ The results of the SAXS measurements, solution structure modeling (using the available X-ray and NMR model coordinates of yeast frataxin), and model fitting to the experimental data are summarized in Fig. 1a and Table 1. Since no concentration effects were observed in the experiments with glycerol, data with the highest concentration were used for analysis and structural modeling (Fig. 1a, iv-vi). The radius of gyration $\left(R_{\mathrm{g}}\right)$, estimated using Guinier approximation, was $1.80 \pm 0.1$ and $2.25 \pm 0.1 \mathrm{~nm}$ for $\mathrm{Yfh} 1_{\mathrm{wt}}$ and Yfh1 $1_{\mathrm{wt}}+$ glycerol, respectively. Given that the ratio $V_{\mathrm{p}} / \mathrm{MW}\left(V_{\mathrm{p}}\right.$ is the Porod volume; $\mathrm{MW}$ is the molecular weight) is expected to be approximately $1: 1.7$, the volume in the absence of glycerol is consistent with the molecular mass of the frataxin monomer $(13.7 \mathrm{kDa})$. In the presence of glycerol, the volume is significantly greater, indicating the presence of higher-order oligomers. Theoretical scattering calculated for the $X$-ray structure of the Y73A variant of yeast frataxin yielded an $R_{\mathrm{g}}$ value of $2.07 \mathrm{~nm}$ (the new X-ray model, see below), while for the NMR structure of $Y$ fh $1_{\mathrm{wt}}$ [Protein Data Bank (PDB) ID: 2GA5], the different conformers yielded $R_{\mathrm{g}}$ values in the range $1.54-1.72 \mathrm{~nm}$. Although these values are close to the $R_{g}$ estimated from the SAXS data for the protein without glycerol, as seen in Fig. 1a (i and ii, Yfh $1_{\mathrm{wt}}$, and iv and $\mathrm{v}, \mathrm{Yfh} 1_{\mathrm{wt}}+$ glycerol), neither the X-ray model nor any of the NMR models provided satisfactory fit to the experimental SAXS data (the corresponding discrepancies $x$ are shown in Table 1). We also estimated the maximum dimensions $\left(D_{\max }\right.$, see Table 1$)$ and the interatomic distance distribution function $p(r)$ of $\mathrm{Yfh}_{\mathrm{wt}}$ in the presence and absence of glycerol using GNOM. ${ }^{32}$ The $p(r)$ for $\mathrm{Yfh}_{\mathrm{wt}}+$ glycerol showed shoulders at larger distances and increase in $D_{\max }$ value (Table 1 ), as compared to $\mathrm{Yfh}_{\mathrm{wt}}$. These features are typical of dynamic systems. ${ }^{33}$

For the interpretation of the SAXS data, we subsequently performed $a b$ initio modeling using the DAMMIN software. Ten $a b$ initio models independently generated using DAMMIN had a mean normalized spatial discrepancy (NSD), as calculated by DAMAVER, in the range 0.54-0.59 and $0.52-0.58$ for the $Y$ fh $1_{\mathrm{wt}}$ data and the $\mathrm{Yfh} 1_{\mathrm{wt}}+$ glycerol data, respectively. The best $a b$ initio model had a fit to the experimental scattering of $x=1.16$ (Table 1). At the next step, higher resolution ab initio models were generated using GASBOR. Twenty GASBOR models had NSD in the range 0.9-0.96, which illustrates that all shapes were essentially similar and that a unique solution had been identified. The best $Y$ fh1 $1_{\mathrm{wt}}$ GASBOR model was selected for rigid-body superposition with the model of the molecule (Fig. 1b).

The NMR model and the X-ray structure suggest that yeast frataxin monomer has a highly flexible Nterminus, which may explain the abovementioned poor agreement of the X-ray and NMR models (PDB ID: 2GA5) with the experimental scattering data. To fit the NMR and X-ray models to the SAXS data, we employed a combination of $a b$ initio and rigid-body modeling approaches to remodel the N-terminus, using the program BUNCH. During this process, the interatomic distances and angles between "dummy" atoms of the $a b$ initio model were constrained to mimic the $\mathrm{C}^{\alpha}$ peptide chain. Ten BUNCH models were generated for the different data sets. The models showed good agreement with the $\mathrm{Yfh}_{\mathrm{wt}}$ scattering data (s ranges from 0.020 to $2.50 \mathrm{~nm}^{-1}$ ), with $\chi$ in the range 1.04-1.40, but rather poor agreement with the Yfh $1_{\mathrm{wt}}+$ glycerol data, with $\chi$ in the range 1.4-2.4 (compare Fig. 1a, ii and v, red curve). Furthermore, assuming that different frataxin conformations exist in solution in equilibrium with each other, we used OLIGOMER to calculate the volume fractions of the separate NMR conformers contributing to the scattering profile. The results showed that only two conformers, \#8 and \#16 (PDB ID: 2GA5), contributed $32 \%$ and $68 \%$ volume fractions, respectively, to the scattering. This further improved the discrepancy of the fitting to the $Y$ fh $1_{w t}$ data, as compared to the lowest-energy NMR conformer or the X-ray structure, but not for the case of the data in the presence of glycerol. This can be seen from the comparison of the discrepancy values in Table 1 and is also shown in Fig. 1a for the data in the absence and presence of glycerol (pink

Table 1. The results of SAXS measurements on the monomeric yeast frataxin in the absence and presence of glycerol

\begin{tabular}{lcccccccccc}
\hline Sample & $R_{\mathrm{g}}(\mathrm{nm})$ & $D_{\max }(\mathrm{nm})$ & $V_{\mathrm{e}}\left(\mathrm{nm}^{3}\right)$ & $V_{\mathrm{p}}\left(\mathrm{nm}^{3}\right)$ & $\chi_{\mathrm{g}}$ & $\chi_{\mathrm{x}}$ & $\chi_{\mathrm{n}}$ & $\chi_{\mathrm{o}}$ & $\chi_{\mathrm{b}}$ & $\chi_{\mathrm{f}}$ \\
\hline Yfh1 $_{\mathrm{wt}}$ & 1.8 & 6.8 & 28 & 24 & 1.16 & 1.19 & 1.16 & 1.03 & 1.04 & 1.02 \\
Yfh1 $_{\mathrm{wt}}+$ glycerol & 2.25 & 7.5 & 32.2 & 44 & 1.36 & 1.2 & 3.26 & 2.53 & 1.4 & 1.01 \\
\hline
\end{tabular}

$R_{\mathrm{g}}, D_{\max }, V_{\mathrm{e}}$, and $V_{\mathrm{p}}$ are radius of gyration, maximum size, excluded volume, and Porod volume, respectively. $\chi_{\mathrm{g}}$, discrepancy of GASBOR $a b$ initio models; $x_{x}$, discrepancy to new X-ray structure; $\chi_{n}$, discrepancy to lowest-energy NMR conformer (model \#1 in 2GA5); $\chi_{\mathrm{b}}$, discrepancy to the BUNCH model; $\chi_{o}$, OLIGOMER discrepancy to 20 NMR conformers and X-ray model; $\chi_{f}$, discrepancy to EOM ensemble model. 
curves, Fig. 1a, iii and vi, respectively). In the latter case, the poor fit of the model to the data is clearly visible in the low-resolution range. We also compared the reference $a b$ initio model generated by GASBOR to the rigid-body model with the lowest discrepancy generated by BUNCH (see Fig. 1b). The superposition showed very good agreement, indicating that both reconstructions converged to a similar solution. It is noteworthy that both $a b$ initio and rigid-body models are likely to represent an average over a set of conformations in solution and that the good agreement between them suggests validity of such models.

To improve the fit for the case of $\mathrm{Yfh}_{\mathrm{wt}}+$ glycerol and quantitatively characterize the ensemble properties of $\mathrm{Yfh}_{\mathrm{wt}}$ and $\mathrm{Yfh} 1_{\mathrm{wt}}+$ glycerol in solution, we applied the ensemble optimization method (EOM) to the measured data sets. The EOM analysis allowed us to further improve the fit to the experimental data sets [see Fig. 1a, iii and vi, green curves, and Table $\left.1\left(\chi_{\mathrm{f}}\right)\right]$. As seen from Fig. 1c, $R_{\mathrm{g}}$ distributions corresponding to $Y \mathrm{fh} 1_{\mathrm{wt}}$ and $\mathrm{Yfh} 1_{\mathrm{wt}}$ + glycerol ensembles show considerable differences. Thus, in the case of Yfh $1_{\mathrm{wt}}$, only a negligible fraction of dimers was selected. If a random pool only contained monomers, the discrepancy did not change as compared to the mixed full pool. On the other hand, the $Y \mathrm{fh} 1_{\mathrm{wt}}+$ glycerol data could only be fitted with more extended monomers and a larger fraction of dimers, as compared to $Y$ fh $1_{\mathrm{wt}}$. The data could not be fitted if a random pool only contained monomers. On the other hand, if only three curves per ensemble were allowed to be selected, dimers were always present in the ensemble and the discrepancy was in the range 1.06-1.07. The average radius of gyration of the EOM ensemble, as seen from Fig. 1c, was found to be close to the $R_{\mathrm{g}}$ calculated by Guinier approximation (1.80 \pm 0.1 and $2.25 \pm 0.1 \mathrm{~nm}$ for $\mathrm{Yfh} 1_{\mathrm{wt}}$ and $\mathrm{Yfh} 1_{\mathrm{wt}}+$ glycerol, respectively; see above).

The results clearly show that $\mathrm{Yfh} 1_{\mathrm{wt}}$ in solution exists in different conformations and that the primary source of the conformational heterogeneity is the high level of flexibility of the N-terminus. Furthermore, while experimental SAXS data for $\mathrm{Yfh}_{\mathrm{wt}}$ can be described with an ensemble model, mainly consisting of monomers, a dynamic monomer-dimer equilibrium exists in the case of $Y f h 1_{w t}+$ glycerol. Hence, the addition of glycerol apparently results in the formation of dimers, which presumably could be considered as the first stage in the oligomerization process of frataxin.

\section{Characterization of the Yfh1 Y73A variant oligomers using SAXS}

Gel-filtration studies have shown that the Yfh1 Y73A variant is eluted as two major fractions, one of lower molecular weight, suggested to represent a trimer, and the second of higher molecular weight, suggested to be a 24-mer. As noted above, the low molecular weight fraction was crystallized and revealed the trimeric frataxin structure, while the high molecular weight fraction was studied by single-particle electron microscopy (EM) reconstruction and revealed a 24-meric particle built up of 8 trimers. ${ }^{22,26}$ For a better understanding of the role of the Y73A replacement on the oligomerization propensity of the protein, we studied the low-molecular-weight fraction using DLS (Fig. 2a) and SAXS (Fig. $2 \mathrm{~b}$ and Table 2). The DLS measurements, analysis of the SAXS data, and structural models generated to fit the data (see Materials and Methods) indeed reveal the presence of several oligomeric species in solution. As seen from Table 2, while the major part of the protein in solution is in monomeric form (around 60\%), there is also a proportion of trimers, hexamers, and dodecamers $(9 \%, 20 \%$, and $11 \%$, respectively). Two different models (based on NMR conformers and kinematic loop modeling) were needed to model the monomers, which confirm the highly flexible nature of the monomeric species. With this pool of monomers and oligomers, the generated models could fit the SAXS data with $\chi^{2}=0.44$, as estimated by the program OLIGOMER. There were no signs of protein aggregation, as shown by the analysis of the Guinier region of the data (Fig. S1a). These results clearly showed that Y73A modification of yeast frataxin results in a higher propensity to form higher-order oligomers, independently of the presence of metals, perhaps as a result of reduced flexibility of the N-terminal region of the protein. During crystallization, the trimeric form is presumably selected as the most stable in the formation of the crystal lattice.

\section{DLS studies of cobalt-induced oligomerization of yeast frataxin}

To further investigate the influence of various factors on the oligomerization of yeast frataxin, we used DLS to study the effects of $\mathrm{Mg}^{2+}, \mathrm{Zn}^{2+}$, and $\mathrm{Co}^{2+}$. The choice of divalent metals was dictated by the available biochemical data, which indicated that ferrous iron induces formation of Yfh1 oligomers. While $\mathrm{Mg}^{2+}$ did not show any visible effects in our experiments, the addition of $\mathrm{Zn}^{2+}$ resulted in aggregation and precipitation of the sample (data not shown). On the other hand, $\mathrm{Co}^{2+}$ appeared to be the most suitable metal and induced the formation of higher-order oligomers in a concentration-dependent fashion. It appears that at least in this respect, Yfh1 and its bacterial homologue CyaY behave similarly in response to metal binding. ${ }^{34}$ Other advantages of using cobalt include its stability, which allows avoiding the formation of metal oxidation products, and an ionic radius, which is similar to that of $\mathrm{Fe}^{2+}$. 
(a)

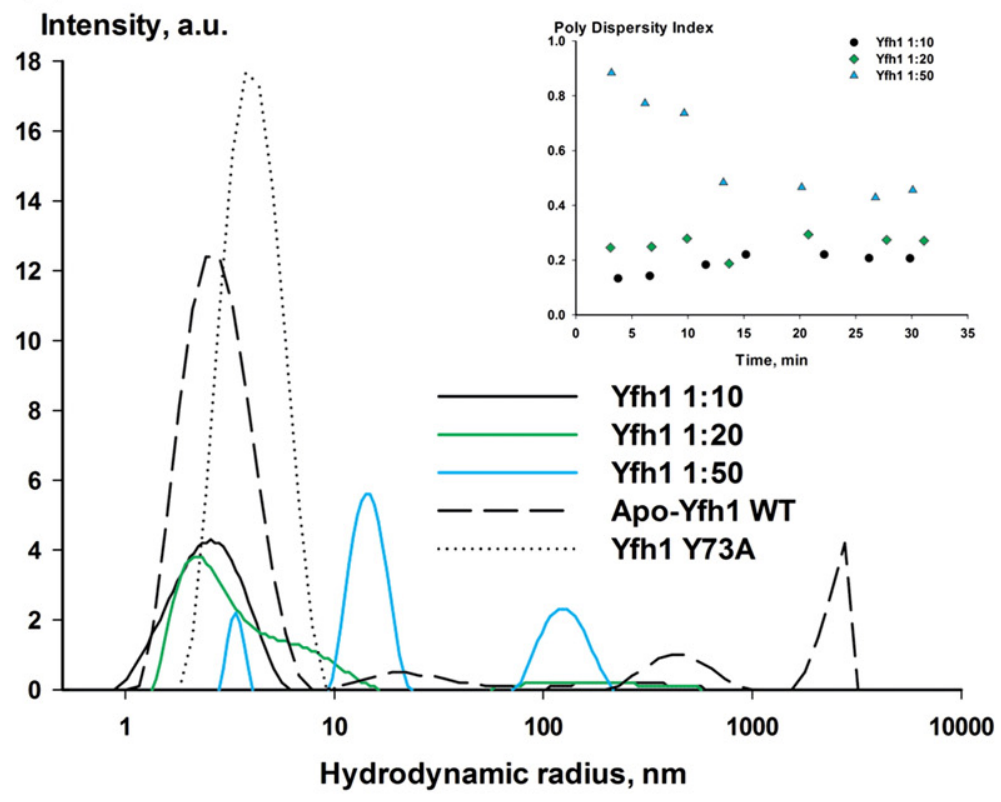

(b)

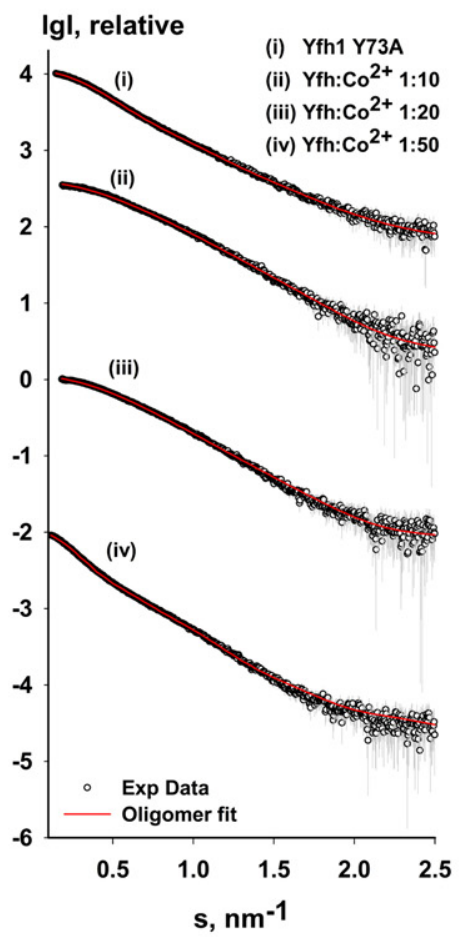

Fig. 2. Oligomerization of yeast frataxin homologue. (a) Sample size distribution (hydrodynamic radius) observed using DLS. Wild-type Yfh1 and the Y73A variant are shown as dashed and dotted lines, respectively; Yfh1: $\mathrm{CoCl}_{2}$ ratios $(1: 10,1: 20$, and 1:50) are shown in black, green, and blue, respectively. The inset shows the time dependence of the polydispersity index after addition of $\mathrm{CoCl}_{2}$. (b) Experimental SAXS profiles (with experimental data shown as black circles) for the Y73A variant of Yfh1 and wild-type Yfh1 in the presence of different amounts of metal, as shown in the figure. The corresponding fit curve is overlaid on each profile (red) (see Table 2 for details). The data are shown to a maximum momentum transfer of $s=2.5 \mathrm{~nm}^{-1}$. See also Fig. S1a for plots of the Guinier region.
The protein-to-metal ratios (Yfh1: $\left.\mathrm{CoCl}_{2}\right)$ in the experiments were optimized to 1:10, 1:20, and 1:50, and the protein concentration was $3 \mathrm{mg} / \mathrm{ml}$. As controls, we also used the Y73A Yfh1 variant, which, as shown above, is prone to the formation of higherorder oligomers, and the wild-type $\mathrm{Yfh} 1_{\mathrm{wt}}$ without metal. For each concentration of $\mathrm{CoCl}_{2}$, light scattering and the respective polydispersity indices were measured several times over a period of $30 \mathrm{~min}$. After approximately $30 \mathrm{~min}$, the polydispersity index was stable, indicating that equilibrium had been reached (Fig. 2a, inset). This incubation time was also used later in our SAXS measurements.

As shown in Fig. 2a, at a $\mathrm{Yfh} 1_{\mathrm{wt}}: \mathrm{CoCl}_{2}$ ratio of $1: 10$, no substantial changes in the DLS profile could be observed, as compared to the protein solution 
Table 2. The combinations of the models with different oligomeric states used for fitting to the experimental data (Fig. 2b) are shown together with their respective distributions in the mixture and the discrepancy $\left(\chi^{2}\right)$

\begin{tabular}{|c|c|c|c|c|c|}
\hline Sample & $R_{g}(\mathrm{~nm})$ & $D_{\max }(\mathrm{nm})$ & Oligomer model & Distribution (\%) & $x^{2}$ \\
\hline Y73A Yfh1 & $3.2 \pm 0.1$ & $11.5 \pm 0.5$ & $1^{\mathrm{a}} / 1^{\mathrm{b}} / 3^{\mathrm{c}} / 6^{\mathrm{d}} / 12^{\mathrm{e}}$ & $36 / 24 / 9 / 20 / 11$ & 0.44 \\
\hline 1:10 Yfh1:Co[II] & $2.3 \pm 0.1$ & $8 \pm 0.5$ & $1^{\mathrm{a}} / 3^{\mathrm{f}} / 6^{\mathrm{d}}$ & $67 / 24 / 9$ & 0.55 \\
\hline 1:20 Yfh1:Co[II] & $2.5 \pm 0.1$ & $9 \pm 0.5$ & $1^{\mathrm{a}} / 3^{\mathrm{f}} / 6^{\mathrm{d}}$ & $54 / 33 / 13$ & 0.55 \\
\hline 1:50 Yfh1:Co[II] & $5.4 \pm 0.1$ & $19 \pm 0.5$ & $1^{\mathrm{a}} / 3^{\mathrm{f}} / 6^{\mathrm{d}} / 42^{\mathrm{g}}$ & $26 / 57 / 6 / 11$ & 0.67 \\
\hline
\end{tabular}

$R_{\mathrm{g}}$ and $D_{\max }$ are radius of gyration and maximum size, respectively.

${ }^{\text {a }}$ NMR conformer(s).

b Kinematic loop model(s).

c Tilted trimer from Rosetta docking.

d Hexamer from EM density docking.

e Dodecamer from EM density docking.

f Trimer with rebuilt N-terminus using Rosetta.

g 42-mer from combining two EM-docked 24-mers and removing two overlapping trimers.

without added metal. Increasing the ratio to 1:20 resulted in tailing of the peak, with a particle size distribution around $2.3 \mathrm{~nm}$. At a $\mathrm{Yfh}_{\mathrm{wt}}: \mathrm{CoCl}_{2}$ ratio of 1:50, three separate peaks with particle size distributions of around $3.5 \mathrm{~nm}, 15 \mathrm{~nm}$, and $125 \mathrm{~nm}$ could be observed. This clearly demonstrates that in a concentration-dependent fashion, $\mathrm{Co}^{2+}$ induces oligomerization of yeast frataxin into larger particles. In the experiments, we found the presence of $100 \mathrm{mM} \mathrm{NaCl}$, to be required for optimal protein stabilization and for preventing aggregation. The presence of salt has previously been reported as monomer stabilizing. ${ }^{35}$ However, in our experiments, this did not prevent oligomer formation in the presence of $\mathrm{Co}^{2+}$.

\section{Characterization of cobalt-induced yeast frataxin oligomers using SAXS}

To further determine the type of oligomers induced by the addition of $\mathrm{Co}^{2+}$, we used SAXS to examine the solutions with the same protein-tometal ratios, as in the DLS experiments (1:10, 1:20, and 1:50). Analysis of the Guinier region (Fig. S1a) confirmed that addition of $\mathrm{Co}^{2+}$ to the samples at these concentrations did not induce aggregation, although the sample with a 1:50 ratio showed signs of polymerization, given the high average $R_{\mathrm{g}}$ value of $5.45 \mathrm{~nm}$ (around $1.8 \mathrm{~nm}$ for the monomeric protein, as described above). A comparison of the calculated average molecular weights, using excluded volume and lysozyme as a standard, revealed an increase with higher concentrations of $\mathrm{Co}^{2+}$. Figure $2 \mathrm{~b}$ and Table 2 show that the data for the 1:10, 1:20, and 1:50 ratios could be fitted with $\chi^{2}$ values of 0.55 , 0.55 , and 0.67 , respectively, given the input we provided from our pool of oligomers and using the program OLIGOMER. Analysis of the volume distributions of oligomer fitting to the respective SAXS profiles showed that as $\mathrm{Co}^{2+}$ concentration increased, so did the volume fraction of frataxin trimer. In other words, $\mathrm{Co}^{2+}$ strongly supports the formation of trimers.
Analysis of the $\mathrm{Co}^{2+}$-induced oligomeric states (Table 2) clearly showed a gradual increase in the percentage of trimers and hexamers and a concomitant decrease in the percentage of monomers, from $67 \%$ at a protein-to-metal ratio of $1: 10$ to $26 \%$ at 1:50. It was also evident that at the 1:50 ratio, oligomers of considerably higher order were formed. Interestingly, while the percentage of trimers increases from $24 \%$ to $57 \%$ when the protein-to-metal ratio changes from 1:10 to 1:50, the number of hexameric species only reaches $9 \%$. This is different from the oligomerization behavior of the Y73A variant, which shows higher percentage of hexameric species, compared to trimers (20\% and 9\%, respectively; Table 2$)$. These results show that the oligomerization behavior of frataxin can be changed relatively easy and that the presence of $\mathrm{Co}^{2+}$ specifically stabilizes the trimers, supporting earlier biochemical data on metalinduced oligomerization of yeast frataxin. ${ }^{36}$

\section{X-ray crystallographic characterization of cobalt binding to yeast frataxin}

The crystals of the Y73A variant of Yfh1, which were produced from the modified crystallization conditions, belonged to space group $122_{1} 3$ with one monomer in the asymmetric subunit and diffracted to $2.9 \AA$ resolution (Table 3 ). As discussed previously, ${ }^{26}$ the monomers are involved in extensive interactions around the channel at the 3-fold axis. Their N-termini form a bridge between the monomers, which adds to the stability of the trimeric structure. The new data allowed the electron density for the complete N-terminal part of the structure to be traced, adding nine residues (V52-V60) to the new model. In the present conformation of the N-terminus, the side chains of E64 and E112 are within hydrogen-bonding distance (3.1 Å) from each other, while the newly added part of the N-terminus seems to be stabilized primarily by van der Waals contacts between side chains within the region V52-S54 and residues from the 
Table 3. X-ray data collection and refinement statistics for the crystal structure of the Y73A variant of Yfh1 with and without $\mathrm{Co}^{2+}$

\begin{tabular}{|c|c|c|}
\hline Data collection & Y73A & Co(II)-soak \\
\hline Beamline & I911-2 & 1911-3 \\
\hline Space group & $I 2{ }_{1} 3$ & $I 2{ }_{1} 3$ \\
\hline Wavelength $(\AA)$ & 1.038 & 1.605 \\
\hline Cell dimensions, $a=b=c(\AA)$ & 121.36 & 121.20 \\
\hline Resolution range $(\AA)$ & $2.96(3.03-2.96)$ & $3.2(3.28-3.2)$ \\
\hline Completeness $(\%)$ & $99.0(99.1)$ & $99.8(99.9)$ \\
\hline$I / \sigma(I)(\%)$ & $22.6(2.5)$ & $25.4(3.1)$ \\
\hline No. of unique reflections & 12,005 (929) & 9507 (705) \\
\hline $\begin{array}{l}R_{\text {merge }}{ }^{\mathrm{a}} \\
\text { Refinement }\end{array}$ & $5.6(65.3)$ & $4.6(49.3)$ \\
\hline$R_{\text {cryst }}\left(R_{\text {free }}\right)^{\mathrm{b}}$ & $0.266(0.292)$ & $0.204(0.256)$ \\
\hline r.m.s.d.bond $(\AA)$ & 0.014 & 0.014 \\
\hline r.m.s.d.angles $\left({ }^{\circ}\right)$ & 1.71 & 1.83 \\
\hline
\end{tabular}

${ }^{\text {a }} R_{\text {merge }}=\sum\left|I_{i}-\langle I\rangle\right| / \sum I$, where $I_{i}$ is an individual intensity measurement and $\langle I\rangle$ is the average intensity for this reflection.

${ }^{\mathbf{b}} R_{\text {cryst }}=\sum\left|F_{\mathrm{o}}-F_{\mathrm{c}}\right| / \sum F_{\mathrm{o}}$, where $F_{\mathrm{o}}$ and $F_{\mathrm{c}}$ are the observed and calculated structure factor amplitudes, respectively. $R_{\text {free }}$ is the same as $R_{\text {cryst }}$ but calculated on $5 \%$ of the data excluded from refinement.

loop between $\beta$-strands beta1 and beta2 (H106 is shown on Fig. 3a).

Analysis of the packing and interactions within the crystal lattice is of interest for our understanding of the interactions that stabilize high-order oligomers. The previous model of frataxin (PDB ID: 2FQL) showed that the trimers within the crystal lattice were packed against each other with the Ntermini from neighboring trimers building an intersection (involving amino acid residues P69, L70, E71, and K72). In the new model, due to the extension of the N-terminal part, we could observe an additional intersection involving amino acid residues Q59, V60, and V61 (Fig. 3b; only V60 is shown). This part of the N-terminus contributes to the stabilization of the interactions between trimers within the crystal lattice (Fig. 3c) and could also stabilize transient monomer-monomer interactions in solution, thus driving the oligomerization towards higher-order oligomers. Upon hexamer formation, as suggested by the models used to fit the SAXS data, this type of interaction between the N-

Fig. 3. The X-ray structure of the Y73A frataxin variant. (a) The interaction of the N-terminal region of one of the subunits with the neighboring subunit. Amino acid side chains are shown as sticks, and a possible hydrogen bond between E64 and E112 is shown as a broken line. (b) The intersection of the N-termini of two monomers belonging to two different trimers within the crystal lattice. (c) Packing of Y73A frataxin trimers, with each trimer colored uniquely. One of the monomers is shown in surface representation for clarity. The N-terminal residues 52-60 are colored yellow and red. (d) Packing of the Y73A variant into hexamers, as suggested by the models of the hexamers used to fit the SAXS scattering data (see Materials and Methods). (a)

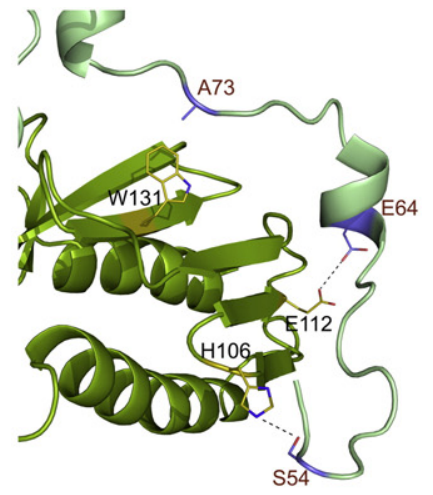

(b)

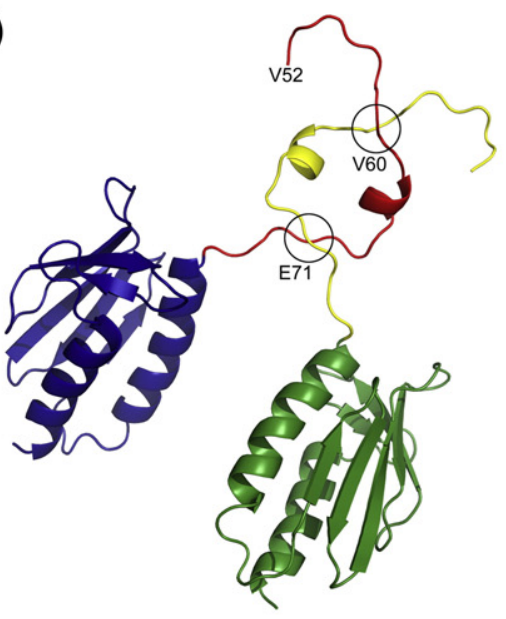

(c)

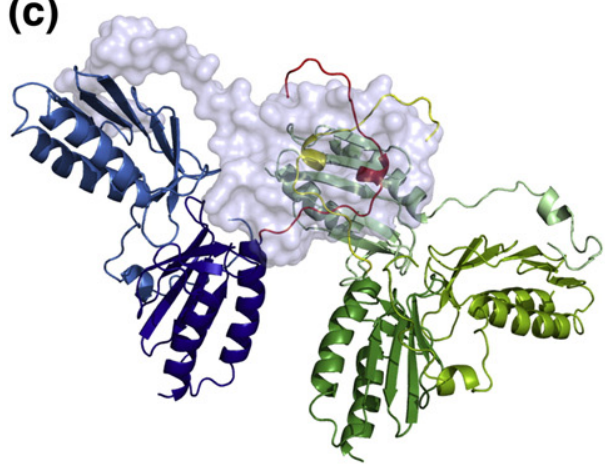

(d)

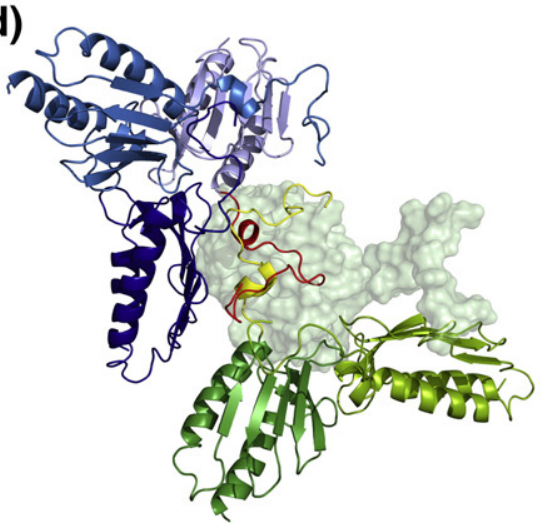


termini is preserved (Fig. 3d). However, in this case, the interacting $\mathrm{N}$-termini have to be in a slightly different conformation in order to avoid a steric clash with neighboring trimers (further discussed in Discussion).

To assess the ability of cobalt to bind to frataxin, we first soaked the crystals of frataxin trimers with $\mathrm{CoCl}_{2}$. X-ray data were collected, and the presence of bound metal in the structure was validated by the examination of the $F_{\mathrm{o}}-F_{\mathrm{c}}$ and the anomalous Fourier difference maps. Our previous crystallographic work showed that the three-dimensional
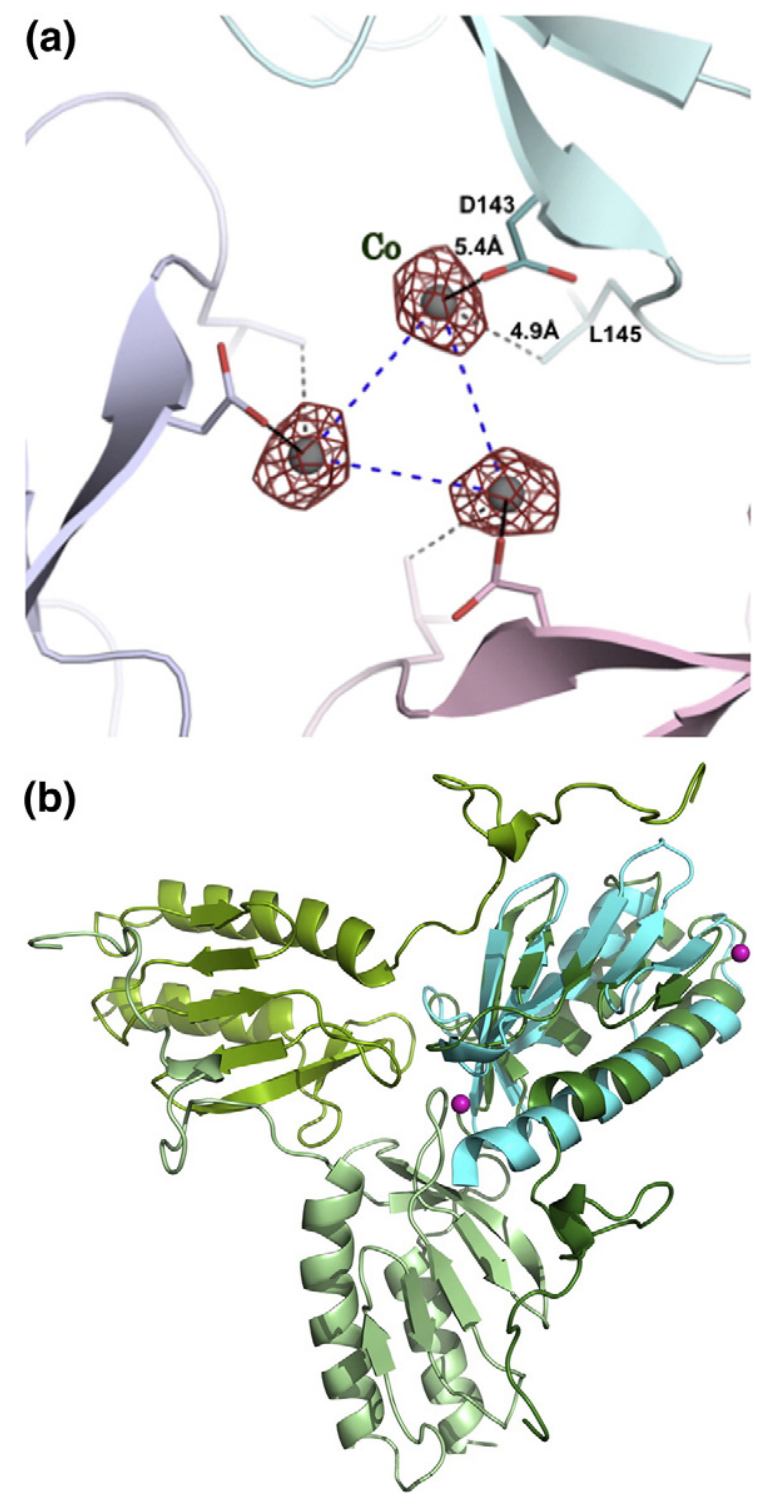

Fig. 4. (a) The $2 F_{\mathrm{o}}-F_{\mathrm{c}}$ electron density map contoured at $1.0 \sigma$ for the cobalt. Three cobalt atoms are located around the 3-fold axis, with 30\% occupancy each. (b) Superposition of the structures of yeast frataxin (green) and its bacterial homologue CyaY (cyan, PDB code 2EFF) showing the positions of the cobalt ions (sphere) in CyaY. structure of frataxin trimers pre-loaded with iron contained a metal bound in the channel at the 3-fold axis of the trimer, ${ }^{26}$ although the resolution of that structure was only $3.5 \AA$. With the current higherresolution data, soaking with cobalt clearly showed a peak in both the $F_{\mathrm{o}}-F_{\mathrm{c}}$ and the anomalous difference density maps at levels of up to $6 \sigma$ and $5 \sigma$, respectively. At the occupancy value of 0.33 , there was no residual density in the $F_{\mathrm{o}}-F_{\mathrm{c}}$ maps, and the $B$-factor of the metal was refined to a value similar to that of the surrounding protein groups. Figure 4a shows three metal ions bound within the channel between the monomers. This mode of binding is different from that which was observed for iron binding in iron-preloaded trimers and suggests that one cobalt ion is shared by the three monomers in the trimer. This also suggests that the affinity of $\mathrm{Co}^{2+}$ to the binding site is lower than that of iron. While the electron density around the bound iron clearly suggested that the metal was hydrated, ${ }^{26}$ the low occupancy of the cobalt and the resolution of the structure would prevent the density of the solvent to be observed. However, the position of the metal at about $5 \AA$ from the invariant D143 close to the wider opening of the channel suggests that some solvent molecules may be bound to the metal. It should be noted that even in the case of the iron-loaded trimer structure, the metal did not show direct interactions with the invariant D143. ${ }^{26}$

\section{Discussion}

In this work, we have studied the effect of three different factors on the oligomerization properties of yeast frataxin Yfh1 and have shown that, depending on the conditions, it may exist in different oligomeric states in solution. Thus, the presence of glycerol facilitated the dimerization of the protein, resulting in dynamic monomer-dimer equilibrium. Glycerol is generally known to contribute to protein stability, by inducing protein compaction and by reducing flexibility. ${ }^{37}$ Taking into account the much higher viscosity of the native environment of proteins, and due to various crowding effects, ${ }^{38,39}$ it would be logical to suggest that the monomeric form of yeast frataxin is rare in vivo. It may also be that in solution, the dimer structure serves as a seed for the growth of higher-order oligomers, since the intertwined interaction between the monomers may be already formed at this stage.

The data obtained from both the Yfh1 $1_{\mathrm{wt}}$ and the Y73A variant show that the N-terminus is highly flexible and that the conformation seen in the X-ray structure of the Y73A trimer is presumably a result of stabilization of the $\mathrm{N}$-terminus by interactions with neighboring monomers as well as by crystal packing interactions. This is in contrast to solution data, which show smaller relative percentage of 
Y73A trimers in solution, as compared to hexamers ( $9 \%$ and $20 \%$, respectively, Table 2 ). This suggests that, in solution, the interactions between trimers within higher-order oligomers may contribute to trimer stabilization, as well as to higher-order oligomer stabilization. The modeling of the hexamer model to fit the SAXS data required some modifications in the conformation of the N-terminal part of the protein, as compared to the conformation in the $\mathrm{X}$-ray structure. One possible hydrogen bond involving the side chains of Y73 and D76 may contribute to the stabilization of this conformation (Fig. 5). Interestingly, in this conformation, W131 appears to be more exposed than in the X-ray structure of the trimer (compare the conformation of the N-terminus in Figs. 3a and 5). This residue has been implicated in interactions with Isu1, the scaffold protein on which iron-sulfur clusters are assembled, ${ }^{40}$ suggesting that higher-order oligomers may be required for the assembly of the $\mathrm{Fe}-\mathrm{S}$ cluster synthesis machinery. ${ }^{41}$

In the presence of $\mathrm{Co}^{2+}$, the trimeric form appears to be dominating. Higher metal concentrations lead to the formation of hexamers and higher-order oligomers. In contrast to the Y73A variant, which preferentially exists in hexameric and higher-order oligomeric forms in solution, the presence of metal appears to be sufficient for the stabilization of the trimeric structure. However, from the present data, it would be difficult to determine whether the higher-order oligomers are directly stabilized by the metal or whether their formation is merely a result of the increasing number of trimers, which may spontaneously assemble into higher-order structures. It is however known that at least in the case of iron, the metal stabilizes higher-order

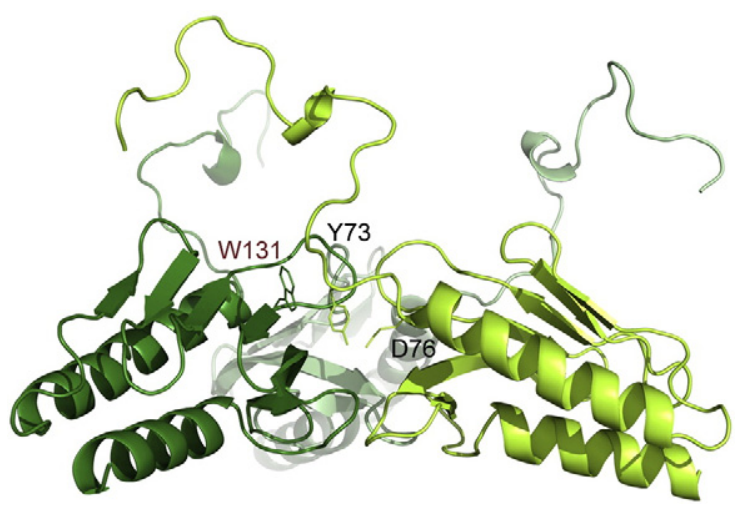

Fig. 5. The conformation of the N-terminal part within the trimer. The trimer that was generated from the X-ray structure to fit the SAXS data (see Materials and Methods section), had a different conformation of the N-terminus (as compared to the X-ray structure). The new conformation exposes residue W131, making it more accessible for the interaction with Isu1. oligomers, while the addition of reducing agents dissolves these complexes. ${ }^{19}$ In the case of the frataxin homologue CyaY from E. coli, it is also known that $\mathrm{Fe}^{2+}$, when added anaerobically, stimulates the formation of tetramers, while larger oligomers are formed in the presence of atmospheric oxygen. ${ }^{21,42}$ It should be noted that the part of the Nterminus that is involved in trimer stabilization in yeast frataxin is absent in CyaY. This suggests that a different type of interaction may be involved in the stabilization of the oligomers in this case. Indeed, when co-crystallized with $\mathrm{Co}^{2+}$, no CyaY trimers can be observed in the crystals (space group $P 3_{2} 21$ ), although one of the metal ions in the structure was coordinated to H58, which belongs to the loop between strands 3 and 4 of the $\beta$-sheet. ${ }^{34}$ As shown in Fig. $4 \mathrm{~b}$, the structurally homologous loop in the crystal structure of the Y73A trimer is located at the channel around the 3 -fold axis. ${ }^{26}$ This position is at approximately $14 \AA$ from the position of the cobalt atom observed in the current study. Earlier reports have indicated that iron binds to the acidic patch at helix 1 and strand 1 of the $\beta$-sheet of frataxin. ${ }^{4-46}$ However, in the present study, no metal density was found within this area.

The present data also demonstrate that the oligomerization behavior of frataxin depends on the amino acid composition of the N-terminus. Thus, the higher propensity of the Y73A variant to form oligomers shows that by a single amino acid replacement in this part of the protein, its properties shift to becoming more like human frataxin. This result, together with the known poor conservation of the amino acid sequence and the length of the Nterminus within the frataxin family, suggests that the oligomerization properties of the protein are different in different species. This may in turn contribute to the variations found in the function of the protein, which could occur even within a single organism. This may have interesting implications for the human protein, which is known to exist in at least four different forms, 78-210, 81-210, 56210, and 42-210. It cannot be excluded that these forms may oligomerize differently depending on iron and external conditions. It should be noted that when we refer to "oligomerization", we distinguish it from the term "aggregation", which is often used in the literature as a synonym of "oligomerization". In structural terms, an oligomer (homo-oligomer or hetero-oligomer) is a structurally defined complex between two or more polypeptide units, with defined (and often conserved) interaction areas. ${ }^{47}$ On the other hand, a protein aggregate usually cannot be easily defined in structural terms since different aggregates do not necessarily have the same structure or number of subunits. Also, the interactions between the subunits within the aggregate are not necessarily the same and may be difficult to describe in detail. 


\section{Materials and Methods}

\section{Protein expression and purification}

The Y73A frataxin variant from S. cerevisiae (residues 52-174 of the yeast frataxin sequence, corresponding to the mature form of the protein ${ }^{48}$ ) was recombinantly expressed in E. coli and purified as described previously. ${ }^{26}$ The wild-type protein was purified as described previously. ${ }^{10}$ Protein concentration was determined from $280 \mathrm{~nm}$ light absorbance with an extinction coefficient $\left(\varepsilon_{280 \mathrm{~nm}}\right)$ of $20,000 \mathrm{M}^{-1} \mathrm{~cm}^{-1}$.

\section{Dynamic light scattering}

DLS measurements were made on a Zetasizer Nano S (Malvern Instruments Ltd.) using a scattering angle of $173^{\circ}$ and a laser working at $633 \mathrm{~nm}$. A ZEN2112 quartz cuvette was used, with $20 \mu \mathrm{l}$ reaction mixture, for each measurement. For the study of metal-induced oligomerization, wild-type yeast frataxin at a concentration of $3 \mathrm{mg} / \mathrm{ml}$ was mixed with a buffer containing $\mathrm{CoCl}_{2}$ to give the final protein:metal molar ratios 1:10, 1:20, and 1:50. In the absence of metal, Yfh $1_{\mathrm{wt}}$ and the $\mathrm{Y} 73 \mathrm{~A}$ variant were measured at $2 \mathrm{mg} / \mathrm{ml}$. All solutions were centrifuged at $14,000 \mathrm{rpm}$ for $10 \mathrm{~min}$ prior to the measurements.

\section{SAXS measurements and modeling}

\section{Data collection and reduction}

SAXS data were collected at beamline I711, MAX-Lab synchrotron, and at the European Molecular Biology Laboratory X33 beamline on the storage ring DORIS III [Deutsches Elektronen-Synchrotron (DESY)]. ${ }^{49}$ Protein buffer consisted of $10 \mathrm{mM}$ Hepes-NaOH and $100 \mathrm{mM}$ $\mathrm{NaCl}$. In separate measurements, $5 \%$ glycerol or 2.2 $22 \mathrm{mM} \mathrm{CoCl}_{2}$ was added to the buffer. Yeast frataxin without glycerol and $\mathrm{CoCl}_{2}$ was measured at a concentration of $10 \mathrm{mg} / \mathrm{ml}$. The data were recorded using a Titan CCD (Oxford Diffraction) detector at a sample-detector distance of $1.5 \mathrm{~m}$ and a wavelength of $1.1 \AA$, covering the range of momentum transfer $0.007<s<0.3 \AA^{-1}$. Yeast frataxin samples in glycerol buffer were measured at $15^{\circ} \mathrm{C}$ at a concentration range of $1.3-9.9 \mathrm{mg} / \mathrm{ml}$. The data were recorded using a 1-M PILATUS detector (DECTRIS) at a sample-detector distance of $2.7 \mathrm{~m}$ and a wavelength of $1.5 \AA$, covering the range of momentum transfer $0.012<s<0.6 \AA^{-P}$. No measurable radiation damage was detected on comparison of four successive time frames with 30-s exposures. The data were averaged after normalization to the intensity of the transmitted beam, and the scattering of the buffer was subtracted using PRIMUS. ${ }^{50}$

Forward scattering $I(0)$ and the radius of gyration $R_{\mathrm{g}}$ were evaluated using the Guinier approximation. ${ }^{51}$ These parameters were also computed from the entire scattering patterns using the program GNOM, ${ }^{32}$ which provides the distance distribution functions $p(r)$ and the maximum particle dimensions $D_{\max }$. Molecular weight estimates were made using lysozyme as a standard or from the excluded volume of the hydrated particle (the Porod volume $V_{\mathrm{p}}$ ), computed as reported by Porod. ${ }^{52}$

Evaluation of the theoretical scattering curves from high-resolution X-ray and NMR structures of frataxin (PDB IDs 2FQL and 2GA5, respectively) and fitting to the experimental scattering data were performed using CRYSOL. ${ }^{53}$ Form factors corresponding to individual NMR conformers and X-ray structure were calculated using the "/nmr" option in the FFMAKER tool from the ATSAS package. ${ }^{54}$ For fitting of the observed scattering curves with weighted combinations of form factors, the program OLIGOMER ${ }^{50}$ was used.

\section{Ab initio modeling}

Low-resolution $a b$ initio models were generated with DAMMIN, ${ }^{55}$ which represents a protein by an assembly of densely packed beads. Simulated annealing was used to build a compact interconnected configuration of beads that fitted the experimental data $I(s)$ to minimize discrepancy:

$$
\chi=\sqrt{\frac{1}{(N-1)} \sum_{j}\left[\frac{\left(I_{\exp }\left(S_{j}\right)-c I_{\text {calc }}\left(S_{j}\right)\right)}{\left(\sigma\left(S_{j}\right)\right)}\right]^{2}}
$$

where $N$ is the number of experimental points; $c$ is a scaling factor; and $I_{\text {exp }}(S), I_{\text {calc }}(S)$, and $\sigma\left(S_{j}\right)$ are the experimental intensity, the calculated intensity, and the experimental error at the momentum transfer $S_{j}$, respectively. An alternative higher-resolution $a b$ initio model, which models the particle in solution as a protein-like assembly of dummy residues, was constructed using GASBOR. ${ }^{56}$ This program represents the internal structure of the molecule more accurately than DAMMIN. Multiple DAMMIN and GASBOR calculations were performed to assess the stability of resulting solutions. Ten to twenty independent reconstructions were performed, and the models were averaged with the program DAMAVER. ${ }^{57}$ The mean NSD between models was in the range $0.537-0.590$ and $0.897-0.962$ for the DAMMIN and GASBOR models, respectively. Low NSD values for the DAMMIN and GASBOR models illustrated that all independently generated shapes were very similar and that a unique solution had been identified.

\section{Combined ab initio and rigid-body modeling}

The N-terminus of frataxin was expected to be flexible in solution. NMR structure determination has shown different conformations for approximately the first 20 amino acids and for the C-proximal loop (residues 93-106). Thus, these regions were remodeled using the program BUNCH. ${ }^{58}$ The starting model generated with PRE_BUNCH consisted of two rigid bodies taken from the lowest-energy NMR conformer (model \#1 in 2GA5), residues 21-92 and 107-123. The positions of rigid bodies were fixed as in the NMR structure. Ten BUNCH models were generated, and the model with the lowest discrepancy was superposed with the $a b$ initio model using SUPCOMB13. 59

\section{Flexibility assessment}

Independently, the SAXS data were analyzed using the $\mathrm{EOM}^{60}$ consisting of two separate programs: RANCH 
(Random Chain) and GAJOE (Genetic Algorithm Judging Optimization of Ensembles). The EOM assumes coexistence of a number of conformations in solution for a given construct in order to fit the experimental SAXS data, providing a quantitative characterization of flexibility and analysis of the size distribution of possible conformers. The X-ray model of frataxin (see below) showed trimers within the crystal lattice, and in addition, the structure of 24-meric oligomers, which was determined from EM, clearly demonstrated that the particles were built up by trimers. ${ }^{22}$ The data presented in this work (see below) also suggest that in the presence of glycerol, a large pool of dimers exists in solution. Thus, it would be expected that the smallest oligomerization unit would consist of interacting dimers, which might serve as a nucleus for the formation of trimers, which in turn will form higher-order oligomers. In order to create a pool of random models, we used independently generated BUNCH models. Three starting models were created with PRE_BUNCH, which consisted of one rigid body from the lowest-energy NMR conformer in 2GA5, residues 21-123. Each starting model corresponded to one of the monomers in the trimer structure of frataxin and was used to independently generate (with different random seed number) $13 \mathrm{BUNCH}$ models. These monomers were then combined with each other model into trimers, using an in-house script in the Perl programming language, resulting in 2197 trimer models with asymmetric N-termini. A similar procedure was used to generate 2000 dimers. In addition, 2000 monomer models were generated using $\mathrm{RANCH}$ with one rigid body (model \#1 in 2GA5, residues 21-123). The final random pool consisted of 6000 models, containing equal fractions of monomers, dimers, and trimers. Theoretical scattering curves were then calculated for each model in the pool using CRYSOL. An intensities master file was generated using the program ONEFILE2 from the EOM package. A Size_list file was created using GAJOE. After generation of the pool and additional files required for EOM, GAJOE was used to select subsets of protein models $(\sim 20)$. The average experimental scattering was calculated for each subset and fitted to experimental SAXS data. Subsets were selected many times in order to minimize the discrepancy. Multiple runs of GAJOE were performed (10 independent runs; for each GAJOE run, the genetic algorithm process was repeated 50 times using the default parameters of the genetic algorithm, i.e., 1000 generations, 50 ensembles of theoretical curves, 20 curves per ensemble, 10 mutations per ensemble, and 20 crossings per generation).

\section{$\mathrm{Co}^{2+}$-dependent oligomerization}

For the study of metal-dependent oligomerization of yeast frataxin, three different Yfh1: $\mathrm{CoCl}_{2}$ ratios $(1: 10,1: 20$, and 1:50) were prepared in a buffer consisting of $10 \mathrm{mM}$ Hepes- $\mathrm{NaOH}$ and $100 \mathrm{mM} \mathrm{NaCl}$, using a protein concentration of $3 \mathrm{mg} / \mathrm{ml}$. The SAXS data were recorded at beamline $I 711^{61}$ at the MAX-Lab synchrotron, using the same setup and procedure as described above. For fitting of the coordinates of the frataxin model to the SAXS data, a pool of yeast frataxin oligomers was created. It consisted of (1) all monomeric NMR conformers (20 models) from 2GA5, (2) 10 variants of the Y73A asymmetric unit (a monomer) where residues 52-73 were remodeled using the kinematic loop modeling protocol ${ }^{62}$ of the Rosetta 3.2 software suite ${ }^{63}$ to account for its flexibility, and (3) eight
Y73A crystallographic trimers that were first docked into the single-particle EM reconstruction of the 24-mer. ${ }^{26}$ Subsequently, the resulting 24-mer particle was stripped of trimers in stepwise fashion, creating 21-, 18-, 15-, 12-, 9-, 6-, and 3-subunit oligomers. In addition, the flexible $\mathrm{N}$ terminus was remodeled, using the conformers from kinematic loop modeling above (2), and all steric clashes with the protein core of neighboring subunits were eliminated. We also created $42-78$ subunit oligomers by combining 18- and 21-subunit oligomers as building blocks. Particles of this type have been observed on negatively stained EM micrographs of the Yfh1 Y73A variant (unpublished data), human frataxin, and horse spleen ferritin. ${ }^{16}$ Subsequently, chains A and B of the crystallographic trimer were used as input to the docking protocol $^{64}$ of Rosetta 3.2 in the "local refine" mode, allowing a maximum of $3 \AA$ translation and $8^{\circ}$ rotation in the creation of 2000 dimer models. Similarly, chains A, B, and $C$ of the crystallographic trimer were used for the creation of 2000 trimer models using symmetry-restrained docking protocols. CRYSOL was used to calculate all the scattering patterns, and OLIGOMER was used to calculate the volume fractions of the components that best fitted the observed scattering curves.

\section{Oligomerization of yeast frataxin $Y 73 A$ variant}

For the study of oligomerization induced by replacement of tyrosine residue 73 with an alanine, we used the same methods as described for metal-dependent oligomerization above, at a concentration of $4 \mathrm{mg} / \mathrm{ml}$ for the low-molecular-weight fraction.

\section{X-ray crystallography}

Initial crystallization conditions were found at the MAX-Lab protein crystallization facility with the JCSGplus screen (Molecular Dimensions Ltd.) setup in Greiner low-profile 96-well plates using the Mosquito robot (TTP Labtech, UK). Well and drop solution volumes of $80 \mu \mathrm{l}$ and $200 \mathrm{nl}$ (100 $\mathrm{nl}$ protein and $100 \mathrm{nl}$ well solution), respectively, were used at $20^{\circ} \mathrm{C}$. Crystals were found in the screen solution containing $1.26 \mathrm{M}\left(\mathrm{NH}_{4}\right)_{2} \mathrm{SO}_{4}, 0.2 \mathrm{M}$ $\mathrm{Li}_{2} \mathrm{SO}_{4}$, and $0.1 \mathrm{M}$ Tris, pH 8.5. Optimization of these conditions yielded crystals between 0.2 and $0.5 \mathrm{~mm}$ in size, which grew after 2 weeks at $15^{\circ} \mathrm{C}$. The crystallization drops were prepared by mixing $2 \mu \mathrm{l}$ well solution containing $1.7 \mathrm{M} \quad\left(\mathrm{NH}_{4}\right)_{2} \mathrm{SO}_{4}, 0.2 \mathrm{M} \quad \mathrm{Li}_{2} \mathrm{SO}_{4}, \quad 4 \%$ $\gamma$-butyrolactone, $0.1 \mathrm{M}$ Tris- $\mathrm{HCl}, \mathrm{pH} 8.5$, and $2 \mu \mathrm{l}$ of protein solution containing $9.2 \mathrm{mg} / \mathrm{ml} \mathrm{Y73A} \mathrm{Yfh1p} \mathrm{in}$ $10 \mathrm{mM}$ Hepes, $\mathrm{pH}$ 7.3. These conditions are different from the earlier published crystallization conditions [0.1 M 2[bis(2-hydroxyethyl)amino]-2-(hydroxymethyl)propane1,3-diol, pH 5.5, $2.0 \mathrm{M}\left(\mathrm{NH}_{4}\right)_{2} \mathrm{SO}_{4}$, and $4 \%(\mathrm{v} / \mathrm{v})$ $\gamma$-butyrolactone].

X-ray data to $2.9 \AA$ resolution were collected at the MAX-Lab synchrotron, beamlines 1911-2 and 1911-3. Prior to data collection, the crystals were flash-frozen in a cryosolution containing $2 \mathrm{M}\left(\mathrm{NH}_{4}\right)_{2} \mathrm{SO}_{4}, 4 \%$-butyrolactone, $20 \%$ glycerol, and $0.1 \mathrm{M}$ Tris- $\mathrm{HCl}, \mathrm{pH}$ 8.5. Due to lowresolution diffraction overloads, data collection was divided into long- and short-exposure sets, with exposure times per image of $30 \mathrm{~s}$ and $10 \mathrm{~s}$, respectively. Both sessions covered $90^{\circ}$ rotation with $0.5^{\circ}$ per image. For 
metal ion soaking, prior to flash-freezing, $4 \mathrm{mM} \mathrm{CoCl}_{2}$ was added to the cryo-solution and the crystals were incubated in this solution for $30 \mathrm{~min}$. XDS software ${ }^{65}$ was used for indexing, integration, scaling, and merging. Phaser software $^{66}$ was used for molecular replacement using the previously determined crystal structure of the Y73A variant of Yfh1 ${ }^{26}$ (PDB ID: 2FQL). Refinement was carried out using alternate runs of the program REFMAC5 ${ }^{67}$ (CCP4 6.0.2 suite ${ }^{68}$ ) each followed by inspection and manual rebuilding of the model using Coot 0.4.1. ${ }^{69}$ For TLS refinement, the whole monomeric unit (residues 52-172) was considered as one TLS group. Splitting the structure into two (52-60 and 61-172) or three (52-60, 61-75 and 76172 ) groups did not result in any improvement in map quality or model geometry. OMIT maps ${ }^{70}$ were used to localize the missing N-terminal residues (52-60), which were subsequently manually built into the density. The mean value for the $B$-factors for the cobalt-free structure and the $\mathrm{Co}^{2+}$ complex was refined to $73 \AA^{2}$ and $96 \AA^{2}$, respectively. For the localization of metal positions, anomalous Fourier maps were calculated using the Bijvoet differences $\left(\mathrm{F}^{+}-\mathrm{F}^{-}\right)$as coefficients. The metal density peaks were clearly visible in both the $F_{\mathrm{o}}-F_{\mathrm{c}}$ the and anomalous difference density maps at levels of up to $6 \sigma$ and $5 \sigma$, respectively. The occupancy of the metal was determined by assigning different values to the occupancy followed by inspection of the difference electron density. At the occupancy value of 0.33 , no residual electron density was present. At this occupancy, the B-factor of the metal was refined to a value similar to that of the neighboring protein groups $\left(79 \AA^{2+}\right)$, which indicates the correctness of the chosen occupancy value. A summary of data collection and refinement statistics is presented in Table 3.

\section{Accession numbers}

Atomic coordinates have been deposited in the PDB with accession numbers 3OEQ and 3OER.

Supplementary materials related to this article can be found online at doi:10.1016/j.jmb.2011.10.034

\section{Acknowledgements}

This project was supported by the Swedish Research Council (Vetenskapsrådet), a MAX IV/ ESS grant from the Natural Science Faculty of Lund University, the Crafoord Foundation, and the Carl Trygger Foundation to S.A.-K. and by grant AG15709 from the National Institute on Aging, National Institutes of Health, to G.I. A.S. and D.S. would like to further acknowledge Bundesministerium für Bildung und Forschung Research Grant SYNC-LIFE (contract number 05K10YEA) and the EU FP7 e-Infrastructure grant WeNMR (contract number 261572). We also thank the European Molecular Biology Laboratory (Hamburg) and Lund University for synchrotron beam time allocation at DESY (Hamburg, Germany) and Max-Lab, respectively. Clement Blanchet and Tomás Plivelic are acknowledged for technical support at beam line X33 (DESY) and I711 (MAX-Lab), respectively.

\section{References}

1. Andrews, S. C., Robinson, A. K. \& RodriguezQuinones, F. (2003). Bacterial iron homeostasis. FEMS Microbiol. Rev. 27, 215-237.

2. Kell, D. B. (2009). Iron behaving badly: inappropriate iron chelation as a major contributor to the aetiology of vascular and other progressive inflammatory and degenerative diseases. BMC Med. Genomics, 2, 2.

3. Campuzano, V., Montermini, L., Molto, M. D., Pianese, L., Cossee, M., Cavalcanti, F. et al. (1996). Friedreich's ataxia: autosomal recessive disease caused by an intronic GAA triplet repeat expansion. Science, 271, 1423-1427.

4. Geoffroy, G., Barbeau, A., Breton, G., Lemieux, B., Aube, M., Leger, C. \& Bouchard, J. P. (1976). Clinical description and roentgenologic evaluation of patients with Friedreich's ataxia. Can. J. Neurol. Sci. 3, 279-286.

5. Harding, A. E. \& Hewer, R. L. (1983). The heart disease of Friedreich's ataxia: a clinical and electrocardiographic study of 115 patients, with an analysis of serial electrocardiographic changes in 30 cases. Q. J. Med. 52, 489-502.

6. Finocchiaro, G., Baio, G., Micossi, P., Pozza, G. \& di Donato, S. (1988). Glucose metabolism alterations in Friedreich's ataxia. Neurology, 38, 1292-1296.

7. Babcock, M., deSilva, D., Oaks, R., DavisKaplan, S., Jiralerspong, S., Montermini, L. et al. (1997). Regulation of mitochondrial iron accumulation by Yfh1p, a putative homolog of frataxin. Science, 276, 1709-1712.

8. Chasteen, N. D. \& Harrison, P. M. (1999). Mineralization in ferritin: an efficient means of iron storage. $J$. Struct. Biol. 126, 182-194.

9. Gakh, O., Adamec, J., Gacy, A. M., Twesten, R. D., Owen, W. G. \& Isaya, G. (2002). Physical evidence that yeast frataxin is an iron storage protein. Biochemistry, 41, 6798-6804.

10. Gakh, O., Park, S., Liu, G., Macomber, L., Imlay, J. A., Ferreira, G. C. \& Isaya, G. (2006). Mitochondrial iron detoxification is a primary function of frataxin that limits oxidative damage and preserves cell longevity. Hum. Mol. Genet. 15, 467-479.

11. Nichol, H., Gakh, O., O'Neill, H. A., Pickering, I. J., Isaya, G. \& George, G. N. (2003). Structure of frataxin iron cores: an X-ray absorption spectroscopic study. Biochemistry, 42, 5971-5976.

12. Yoon, T. \& Cowan, J. A. (2003). Iron-sulfur cluster biosynthesis. Characterization of frataxin as an iron donor for assembly of [2Fe-2S] clusters in ISU-type proteins. J. Am. Chem. Soc. 125, 6078-6084.

13. Lesuisse, E., Santos, R., Matzanke, B. F., Knight, S. A. B., Camadro, J. M. \& Dancis, A. (2003). Iron use for haeme synthesis is under control of the yeast frataxin homologue (Yfh1). Hum. Mol. Genet. 12, 879-889.

14. Bulteau, A. L., O'Neill, H. A., Kennedy, M. C., IkedaSaito, M., Isaya, G. \& Szweda, L. I. (2004). Frataxin acts as an iron chaperone protein to modulate mitochondrial aconitase activity. Science, 305, 242-245.

15. Koutnikova, H., Campuzano, V. \& Koenig, M. (1998). Maturation of wild-type and mutated frataxin by the 
mitochondrial processing peptidase. Hum. Mol. Genet. 7, 1485-1489.

16. Cavadini, P., O'Neill, H. A., Benada, O. \& Isaya, G. (2002). Assembly and iron-binding properties of human frataxin, the protein deficient in Friedreich ataxia. Hum. Mol. Genet. 11, 217-227.

17. Schmucker, S., Argentini, M., Carelle-Calmels, N., Martelli, A. \& Puccio, H. (2008). The in vivo mitochondrial two-step maturation of human frataxin. Hum. Mol. Genet. 17, 3521-3531.

18. Gakh, O., Bedekovics, T., Duncan, S. F., Smith, D. Y.t., Berkholz, D. S. \& Isaya, G. (2010). Normal and Friedreich ataxia cells express different isoforms of frataxin with complementary roles in iron-sulfur cluster assembly. J. Biol. Chem. 285, 38486-38501.

19. Park, S., Gakh, O., O'Neill, H. A., Mangravita, A., Nichol, H., Ferreira, G. C. \& Isaya, G. (2003). Yeast frataxin sequentially chaperones and stores iron by coupling protein assembly with iron oxidation. J. Biol. Chem. 278, 31340-31351.

20. O'Neill, H. A., Gakh, O., Park, S., Jin, C., Mooney, S. M., Sampson, M. et al. (2005). Assembly of human frataxin is a mechanism for detoxifying redox-active iron. Biochemistry, 44, 537-545.

21. Bou-Abdallah, F., Adinolfi, S., Pastore, A., Laue, T. M. \& Chasteen, N. D. (2004). Iron binding and oxidation kinetics in frataxin CyaY of Escherichia coli. J. Mol. Biol. 341, 605-615.

22. Schagerlof, U., Elmlund, H., Gakh, O., Nordlund, G., Hebert, H., Lindahl, M. et al. (2008). Structural basis of the iron storage function of frataxin from singleparticle reconstruction of the iron-loaded oligomer. Biochemistry, 47, 4948-4954.

23. Knight, S. A. B., Sepuri, N. B. V., Pain, D. \& Dancis, A. (1998). Mt-Hsp70 homolog, Ssc2p, required for maturation of yeast frataxin and mitochondrial iron homeostasis. J. Biol. Chem. 273, 18389-18393.

24. Voisine, C., Schilke, B., Ohlson, M., Beinert, H., Marszalek, J. \& Craig, E. A. (2000). Role of the mitochondrial Hsp70s, Ssc1 and Ssq1, in the maturation of Yfh1. Mol. Cell. Biol. 20, 3677-3684.

25. O'Neill, H. A., Gakh, O. \& Isaya, G. (2005). Supramolecular assemblies of human frataxin are formed via subunit-subunit interactions mediated by a nonconserved amino-terminal region. J. Mol. Biol. 345, 433-439.

26. Karlberg, T., Schagerlof, U., Gakh, O., Park, S., Ryde, U., Lindahl, M. et al. (2006). The structures of frataxin oligomers reveal the mechanism for the delivery and detoxification of iron. Structure, 14, 1535-1546.

27. Svergun, D. I. \& Mertens, H. D. T. (2010). Structural characterization of proteins and complexes using small-angle X-ray solution scattering. J. Struct. Biol. 172, 128-141.

28. Verstraete, K., Vandriessche, G., Januar, M., Elegheert, J., Shkumatov, A. V., Desfosses, A. et al. (2011). Structural insights into the extracellular assembly of the hematopoietic Flt3 signaling complex. Blood, 118, 60-68.

29. Svergun, D. I., Mylonas, E., Hascher, A., Bernado, P., Blackledge, M. \& Mandelkow, E. (2008). Domain conformation of tau protein studied by solution small-angle X-ray scattering. Biochemistry, 47, 10345-10353.
30. Shkumatov, A. V., Chinnathambi, S., Mandelkow, E. \& Svergun, D. I. (2011). Structural memory of natively unfolded tau protein detected by small-angle X-ray scattering. Proteins: Struct. Funct. Bioinformat. 79, 2122-2131.

31. Pettersen, E. F., Goddard, T. D., Huang, C. C., Couch, G. S., Greenblatt, D. M., Meng, E. C. \& Ferrin, T. E. (2004). UCSF Chimera-a visualization system for exploratory research and analysis. J. Comput. Chem. 25, 1605-1612.

32. Svergun, D. I. (1992). Determination of the regularization parameter in indirect-transform methods using perceptual criteria. J. Appl. Crystallogr. 25, 495-503.

33. Bernado, P. (2010). Effect of interdomain dynamics on the structure determination of modular proteins by small-angle scattering. Eur. Biophys. J. 39, 769-780.

34. Pastore, C., Franzese, M., Sica, F., Temussi, P. \& Pastore, A. (2007). Understanding the binding properties of an unusual metal-binding protein-a study of bacterial frataxin. FEBS J. 274, 4199-4210.

35. Adinolfi, S., Trifuoggi, M., Politou, A. S., Martin, S. \& Pastore, A. (2002). A structural approach to understanding the iron-binding properties of phylogenetically different frataxins. Hum. Mol. Genet. 11, 1865-1877.

36. Adamec, J., Rusnak, F., Owen, W. G., Naylor, S., Benson, L. M., Gacy, A. M. \& Isaya, G. (2000). Irondependent self assembly of recombinant yeast frataxin: implications for Friedreich ataxia. Am. J. Hum. Genet. 67, 549-562.

37. Vagenende, V., Yap, M. G. \& Trout, B. L. (2009). Mechanisms of protein stabilization and prevention of protein aggregation by glycerol. Biochemistry, 48, 11084-11096.

38. Ralston, G. B. (1990). Effects of crowding in protein solutions. J. Chem. Educ. 67, 857-860.

39. Minton, A. P. (2000). Implications of macromolecular crowding for protein assembly. Curr. Opin. Struct. Biol. 10, 34-39.

40. Foury, F., Leidgens, S. \& De Smet, S. (2010). Frataxin interacts with Isu1 through a conserved tryptophan in its beta-sheet. Hum. Mol. Genet. 19, 276-286.

41. Li, H. Q., Gakh, O., Smith, D. Y. \& Isaya, G. (2009). Oligomeric yeast frataxin drives assembly of core machinery for mitochondrial iron-sulfur cluster synthesis. J. Biol. Chem. 284, 21971-21980.

42. Layer, G., Ollagnier-de Choudens, S., Sanakis, Y. \& Fontecave, M. (2006). Iron-sulfur cluster biosynthesis-characterization of Escherichia coli CyaY as an iron donor for the assembly of [2Fe-2S] clusters in the scaffold IscU. J. Biol. Chem. 281, 16256-16263.

43. Huang, J., Dizin, E. \& Cowan, J. A. (2008). Mapping iron binding sites on human frataxin: implications for cluster assembly on the ISUFe-S cluster scaffold protein. J. Biol. Inorg. Chem. 13, 825-836.

44. Nair, M., Adinolfi, S., Pastore, C., Kelly, G., Temussi, P. \& Pastore, A. (2004). Solution structure of the bacterial frataxin ortholog, CyaY: mapping the iron binding sites. Structure, 12, 2037-2048.

45. He, Y. N., Alam, S. L., Proteasa, S. V., Zhang, Y., Lesuisse, E., Dancis, A. \& Stemmler, T. L. (2004). Yeast frataxin solution structure, iron binding, and ferrochelatase interaction. Biochemistry, 43, 16254-16262. 
46. Cook, J. D., Bencze, K. Z., Jankovic, A. D., Crater, A. K., Busch, C. N., Bradley, P. B. et al. (2006). Monomeric yeast frataxin is an iron-binding protein. Biochemistry, 45, 7767-7777.

47. Imperiali, B. \& Ali, M. H. (2005). Protein oligomerization: how and why. Bioorg. Med. Chem. 13, 5013-5020.

48. Branda, S. S., Cavadini, P., Adamec, J., Kalousek, F., Taroni, F. \& Isaya, G. (1999). Yeast and human frataxin are processed to mature form in two sequential steps by the mitochondrial processing peptidase. J. Biol. Chem. 274, 22763-22769.

49. Roessle, M. W., Klaering, R., Ristau, U., Robrahn, B., Jahn, D., Gehrmann, T. et al. (2007). Upgrade of the small-angle X-ray scattering beamline X33 at the European Molecular Biology Laboratory, Hamburg. J. Appl. Crystallogr. 40, S190-S194.

50. Konarev, P. V., Volkov, V. V., Sokolova, A. V., Koch, M. H. J. \& Svergun, D. I. (2003). PRIMUS: a Windows PC-based system for small-angle scattering data analysis. J. Appl. Crystallogr. 36, 1277-1282.

51. Guinier, A. (1939). La diffraction des rayons X aux très petits angles: application à l'étude de phénomènes ultramicroscopiques. Thèse, Masson et cie, Univ. de Paris.

52. Porod, G. (1982). General theory. In Small Angle X-Ray Scattering (Glatter, O. \& Kratky, O., eds), pp. 17-51, Academic Press, London, UK.

53. Svergun, D., Barberato, C. \& Koch, M. H. J. (1995). CRYSOL-a program to evaluate $\mathrm{x}$-ray solution scattering of biological macromolecules from atomic coordinates. J. Appl. Crystallogr. 28, 768-773.

54. Petoukhov, M. V., Konarev, P. V., Kikhney, A. G. \& Svergun, D. I. (2007). ATSAS 2.1—towards automated and web-supported small-angle scattering data analysis. J. Appl. Crystallogr. 40, S223-S228.

55. Svergun, D. I. (1999). Restoring low resolution structure of biological macromolecules from solution scattering using simulated annealing. Biophys. J. 76, 2879-2886.

56. Svergun, D. I., Petoukhov, M. V. \& Koch, M. H. (2001). Determination of domain structure of proteins from X-ray solution scattering. Biophys. J. 80, 2946-2953.

57. Volkov, V. V. \& Svergun, D. I. (2003). Uniqueness of $\mathrm{ab}$ initio shape determination in small-angle scattering. J. Appl. Crystallogr. 36, 860-864.
58. Petoukhov, M. V. \& Svergun, D. I. (2005). Global rigid body modeling of macromolecular complexes against small-angle scattering data. Biophys. J. 89, 1237-1250.

59. Kozin, M. B. \& Svergun, D. I. (2001). Automated matching of high- and low-resolution structural models. J. Appl. Crystallogr. 34, 33-41.

60. Bernado, P., Mylonas, E., Petoukhov, M. V., Blackledge, M. \& Svergun, D. I. (2007). Structural characterization of flexible proteins using small-angle X-ray scattering. J. Am. Chem. Soc. 129, 5656-5664.

61. Knaapila, M., Svensson, C., Barauskas, J., Zackrisson, M., Nielsen, S. S., Toft, K. N. et al. (2009). A new smallangle X-ray scattering set-up on the crystallography beamline I711 at MAX-lab. J. Synchrotron Radiat. 16, 498-504.

62. Mandell, D. J., Coutsias, E. A. \& Kortemme, T. (2009). Sub-angstrom accuracy in protein loop reconstruction by robotics-inspired conformational sampling. Nat. Methods, 6, 551-552.

63. Leaver-Fay, A., Tyka, M., Lewis, S. M., Lange, O. F., Thompson, J., Jacak, R. et al. (2011). ROSETTA3: an object-oriented software suite for the simulation and design of macromolecules. Methods Enzymol. 487, 545-574.

64. Andre, I., Bradley, P., Wang, C. \& Baker, D. (2007). Prediction of the structure of symmetrical protein assemblies. Proc. Natl Acad. Sci. USA, 104, 17656-17661.

65. Kabsch, W. (2010). Xds. Acta Crystallogr., Sect. D: Biol. Crystallogr. 66, 125-132.

66. McCoy, A. J., Grosse-Kunstleve, R. W., Adams, P. D., Winn, M. D., Storoni, L. C. \& Read, R. J. (2007). Phaser crystallographic software. J. Appl. Crystallogr. 40, 658-674.

67. Murshudov, G. N., Vagin, A. A. \& Dodson, E. J. (1997). Refinement of macromolecular structures by the maximum-likelihood method. Acta Crystallogr., Sect. D: Biol. Crystallogr. 53, 240-255.

68. Dodson, E. J., Winn, M. \& Ralph, A. (1997). Collaborative Computational Project, number 4: providing programs for protein crystallography. Methods Enzymol. 277, 620-633.

69. Emsley, P., Lohkamp, B., Scott, W. G. \& Cowtan, K. (2010). Features and development of Coot. Acta Crystallogr., Sect. D: Biol. Crystallogr. 66, 486-501.

70. Bhat, T. N. (1988). Calculation of an OMIT map. J. Appl. Crystallogr. 21, 279-281. 